Pacific Journal of Mathematics

AN ACTION OF THE AUTOMORPHISM GROUP OF A
COMMUTATIVE RING ON ITS BRACER GROUP 


\title{
AN ACTION OF THE AUTOMORPHISM GROUP OF A COMMUTATIVE RING ON ITS BRAUER GROUP
}

\author{
F. R. DEMEYeR
}

\begin{abstract}
An action of the automorphism group of a commutative ring on its Brauer group is given. The action is characterized cohomologically. Relations with the Teichmuller cocycle map and the Schur subgroup are pointed out.
\end{abstract}

In [13] G. J. Janusz gave an action of the automorphism group of a field $K$ on its Brauer group $B(K)$. For number fields he characterized this action in terms of Hasse invariants and applied his results to the problem of the existence of an outer automorphism of the rational group algebra of a finite group.

Here we give an action of the automorphism group of a commutative ring $R$ on its Brauer group $B(R)$ and describe the action cohomologically. Let $A$ be an Azumaya $R$-algebra and let $\sigma$ be an automorphism of $R$. Define a new $R$-algebra ${ }_{\sigma} A$ by letting $A={ }_{\sigma} A$ as rings and with $R$-module action given by $r * a=\sigma^{-1}(r) a$ for $r \in R$, $x \in A$ where multiplication on the right is in $A$. Proposition 2 is the assertion that the correspondence $A \rightarrow{ }_{\sigma} A$ induces an action of the group Aut $(R)$ of automorphisms of $R$ on $B(R)$.

Let $L$ be a finite Galois field extension of $K$ with finite Galois group $G$ and let Aut $(K: L)$ be the group of automorphisms of $K$ which can be extended to $L$. In [11] S. Eilenberg and S. MacLane gave an action of $\operatorname{Aut}(K: L)$ on $H^{n}\left(G, L^{*}\right)$ for $n \geqq 0$. This action corresponds under the natural identification between $B(L / K)$ and $H^{2}\left(G, L^{*}\right)$ with Janusz's action on $B(L / K)$. For a commutative ring $R, B(R)$ is given as the torsion subgroup of $H_{e t}^{2}(R, U)$ [12] and $H_{e t}^{2}(R, U)$ is a limit of Amitsur cohomology groups [17]. For a faithfully flat commutative extension $S$ of $R$ we give an action of Aut $(R: S)$ on $H^{n}(S / R, U)$ (Amitsur cohomology) and show this action commutes with the natural homomorphism given, for example, in [17] from $B(R)$ into $H_{e t}^{2}(R, U)$. We study the problem of extending an automorphism from $R$ to an $R$-algebra $A$ and its relation to normal algebras and the Teichmuller cocycle map. We show that if $K$ is a field of characteristic $=0$ then Aut $(K)$ must always leave the Schur subgroup of $B(K)$ invariant, and we calculate some examples. Throughout all unexplained terminology and notation will be as in [14]. I would like to thank G. J. Janusz, D. Saltman, and D. Zelinsky for helpful remarks.

1. Let $R$ denote a commutative ring, $\sigma \in \operatorname{Aut}(R)$, and let $M$ be 
an $R$-module. Form the new $R$-module ${ }_{\sigma} M$ which is equal to $M$ as an abelian group and with $R$-action given by $r * m=\sigma^{-1}(r) m$ for all $r \in R, m \in M$ where multiplication on the right-hand side is in $M$. This action is well known, see for example [9]. If $A$ is an $R$-algebra then ${ }_{\sigma} A$ is the $R$-algebra equal to $A$ as a ring and ${ }_{\sigma} A$ as an $R$ module.

Lemma 1. Let $A$ and $B$ be $R$-algebras and $M, N$ be $R$-modules. Let $\sigma \in \operatorname{Aut}(R)$, then

(a) ${ }_{\sigma}(A \otimes B) \cong{ }_{\sigma} A \otimes{ }_{\sigma} B$.

(b) ${ }_{\sigma} \operatorname{Hom}_{R}(M, N) \cong \operatorname{Hom}_{R}\left({ }_{\sigma} M,{ }_{\sigma} N\right)$.

(c) $M$ is an R-progenerator if and only if ${ }_{\sigma} M$ is an $R$ progenerator.

(d) $A$ is a separable $R$-algebra if and only if ${ }_{\sigma} A$ is a separable $R$-algebra.

(e) If $\sigma$ induces an automorphism $\bar{\sigma}$ of $R .1$ then $A \cong{ }_{\sigma} A$ as $R$-algebras if and only if $\bar{\sigma}$ extends to a ring automorphism of $A$.

Proof. The isomorphisms in (a) and (b) are the identity map, (c) is well known (p. 115 of [9]).

For (d) the separability of $A$ over $R$ is equivalent to the existence of an idempotent $e \in A \otimes A, \quad e=\sum_{i=1}^{n} a_{i} \otimes b_{i}$, such that $\sum_{i=1}^{n} a_{i} b_{i}=1$ and $(1 \otimes x-x \otimes 1) e=0$ for all $x \in A$. Let $e_{\sigma}=\sum_{i=1}^{n} a_{i} \otimes$ $b_{i}$ in ${ }_{\sigma} A \otimes{ }_{\sigma} A^{0}$, then we still have $\sum_{i=1}^{n} a_{i} b_{i}=1$. Let $\phi:{ }_{\sigma} A \otimes{ }_{\sigma} A^{0} \rightarrow$ $A \otimes A^{0}$ by $\phi(x \otimes y)=x \otimes y$. Since $A \otimes A^{0}={ }_{\sigma}\left(A \otimes A_{0}\right)$ as rings it follows from (a) that $\phi$ is a ring isomorphism. For any $x \in \in_{\sigma} A$ we have

$$
\begin{aligned}
(x \otimes 1) e=\sum_{i=1}^{n} x a_{i} \otimes b_{i} \stackrel{\phi}{\longrightarrow} \sum_{i=1}^{n} x a_{i} \otimes b_{i}=\sum_{i=1}^{n} a_{i} \otimes b_{i} x \stackrel{\phi^{-1}}{\longrightarrow} \sum_{i=1}^{n} a_{i} \otimes b_{i} x \\
=(1 \otimes x) e .
\end{aligned}
$$

This proves (d).

For (e) assume $A \cong{ }_{\sigma} A$ and let $f:{ }_{\sigma} A \rightarrow A$ be the given $R$-algebra isomorphism. Define $\tau$ by $\tau(a)=f(a)$ for all $a \in A\left(A={ }_{\sigma} A\right.$ as rings). Then $\tau \in \operatorname{Aut}(A)$ and for any $r \in R, \tau(r \cdot 1)=f(r \cdot 1)=f(\sigma(r) * 1)=$ $\sigma(r) \cdot f(1)=\bar{\sigma}(r 1)$. Conversely, assume there is an element $\tau \epsilon$ Aut $(A)$ extending $\bar{\sigma}$. Define $f:{ }_{\sigma} A \rightarrow A$ by $f(a)=\tau(a)$ for all $a \in A$. For any $r \in R$ and $a \in{ }_{\sigma} A$ we have $f(r * a)=f\left(\sigma^{-1}(r) \cdot a\right)=\tau\left(\sigma^{-1}(r) \cdot a\right)=$ $\tau \bar{\sigma}^{-1}(r \cdot 1) \cdot \tau(a)=r \cdot \tau(a)=r \cdot f(a)$. It follows that $f$ is an $R$-isomorphism.

We have let Aut $(R: A)$ be the group of all automorphisms of $R$ which can be extended to $A$, it follows from (e) that Aut $(R: A)=$ $\left\{\sigma \in \operatorname{Aut}(R) \mid A \cong{ }_{\sigma} A\right.$ as $R$-algebras $\}$. If $A$ is a faithful $R$-algebra and $\mathscr{G}$ is the group of all automorphisms of $A$ sending $R$ into itself 
it also follows from (e) that $\operatorname{Aut}_{R}(A)$ is a normal subgroup of $\mathscr{G}$ with factor group Aut $(R: A)$.

If $A$ is an Azumaya (=central separable) $R$-algebra let $|A|$ denote the class of $A$ in $B(R)$.

Proposition 2. Let $R$ be a commutative ring, $\sigma \in$ Aut $(R)$, and $A$ an Azumaya $R$-algebra. The correspondence $A \rightarrow{ }_{\sigma} A$ induces an action $\sigma \cdot|A|={ }_{\sigma} A \mid$ of Aut $(R)$ as a group of Automorphism of $B(R)$.

Proof. Since Center $(A)=$ Center $\left.{ }_{\sigma} A\right)$ it follows from Lemma 1 (d) that ${ }_{\sigma} A$ is an Azumaya $R$-algebra if $A$ is an Azumaya $R$-algebra. If $P$ is an $R$-progenerator then ${ }_{\sigma} P$ is an $R$-progenerator by Lemma 1(c). From Lemma 1(a) and (b) we have ${ }_{\sigma}\left(A \otimes \operatorname{Hom}_{R}(P, P)\right) \cong{ }_{\sigma} A \otimes$ $\operatorname{Hom}_{R}\left({ }_{\sigma} P,{ }_{\sigma} P\right)$ so the given action on $B(R)$ is well defined. By Lemma 1(a) the action of $\sigma$ on $B(R)$ is a homomorphism of $B(R)$. If $\tau \in \operatorname{Aut}(R)$ then ${ }_{\sigma \tau} A \cong{ }_{\sigma}\left({ }_{r} A\right)$ as $R$-algebras by $\phi$ where $\phi(\alpha)=a$. Thus the inverse of $\sigma$ is $\sigma^{-1}$ and $\sigma$ acts as an automorphism of $B(R)$. Also, $\sigma(\tau \cdot|A|)=\sigma \tau \cdot|A|$. It follows that $\operatorname{Aut}(R)$ acts as a group of automorphisms of $B(R)$.

Let $S, T$ be commutative, faithfully flat $R$-algebras, let $\sigma \in$ Aut $(R)$ and let $f: S \rightarrow T$ be an extension of $\sigma$. We show $f$ induces an isomorphism from $H^{n}(S / R, F)$ to $H^{n}(T / R, F$ ) (Amitsur cohomo$\operatorname{logy}$ ) when $F$ is the units functor denoted $U$ or the functor Pic which associates to a commutative ring its group of isomorphism classes of invertible projective modules. Then we show that if $g$ is another extension of $\sigma$ from $S$ to $T$ then $g$ induces the same isomorphism on cohomology groups $f$ does. Notation is as on pg. 119 of [14]. Let $f^{n}: S^{n} \rightarrow S^{n}$ by $f^{n}\left(s_{1} \otimes \cdots \otimes s_{n}\right)=f\left(s_{1}\right) \otimes \cdots \otimes f\left(s_{n}\right)$ for all $s_{i} \in S$. Since $\left.f\right|_{R} \in \operatorname{Aut}(R)$ it follows that $f^{n}$ is a well-defined ring isomorphism which extends $\sigma$. The restriction of $f^{n}$ to $U\left(S^{n}\right)$ is a group isomorphism from $U\left(S^{n}\right)$ to $U\left(T^{n}\right)$ which we also denote $f^{n}$. Let $u \in U\left(S^{n}\right)$ and write $u=\sum_{i=1}^{m} s_{i, 1} \otimes \cdots \otimes s_{i, n}$ with $s_{i j} \in S$. Then

$$
\begin{aligned}
\Delta_{n-1}\left(f^{n}(u)\right) & =\Delta_{n-1}\left(\sum_{i=1}^{m} f\left(s_{i, 1}\right) \otimes \cdots \otimes f\left(s_{i, n}\right)\right) \\
& =\sum_{i=1}^{m} \sum_{j=1}^{n+1}(-1)^{j-1} \varepsilon_{j}\left(f\left(s_{i, 1}\right) \otimes f\left(s_{i, n}\right)\right) \\
& =f^{n+1}\left(\sum_{j=1}^{n+1} \sum_{i=1}^{m}(-1)^{j-1} \varepsilon_{j}\left(s_{i, 1} \otimes \cdots \otimes s_{i, n}\right)\right) \\
& =f^{n+1} \Delta_{n-1}(u) .
\end{aligned}
$$

It follows that $f$ induces an isomorphism from $H^{n}(S / R, U)$ to $H^{n}(T / R, U)$ by $v \rightarrow f^{n+1}(v)$ for any $n$-cocycle $v$. We denote the 
induced isomorphism by $f_{*}^{n}$. If $\tau \in \operatorname{Aut}(R)$ and $g$ is an extension of $\tau$ from $T$ to another commutative faithfully flat $R$-algebra $W$ then $g_{*}^{n} f_{*}^{n}=(g f)_{*}^{n}$ since this is the case on the cocycle level. Thus if $g$ is another extension of $\sigma$ to an isomorphism from $S$ to $T$ then $f=g\left(g^{-1} f\right)$ and $g^{-1} f \in \operatorname{Aut}_{R}(S)$. It is a result of Amitsur (see, for example, 3.2 of [2]) that $\left(g^{-1} f\right)_{*}^{n}$ is the identity on $H^{n}(S / R, U)$ so $f_{*}^{n}=g_{*}^{n}$. Thus $\sigma$ induces an isomorphism from $H^{n}(S / R, U)$ to $H^{n}(T / R, U)$. In particular, it follows that we have defined an action of $\operatorname{Aut}(R: S)$ on $H^{n}(S / R, U)$ for all $n \geqq 0$.

Let $S$ be an étale $R$-algebra and let $\sigma \in \operatorname{Aut}(R)$. Then ${ }_{\sigma} S$ is étale and $f: S \rightarrow{ }_{\sigma} S$ by $f(s)=s$ for all $s \in S$ is an extension of $\sigma$. Thus $\sigma$ sends elements of $H^{n}(S / R, U)$ to elements of $H^{n}\left({ }_{\sigma} S / R, U\right)$. From what we have seen it is routine to check that Aut $(R)$ acts on $\lim H^{n}(S / R, U)$ where the limit is taken over all étale extensions $S$ of $R$. Thus Aut $(R)$ acts on $H_{e t}^{n}(R, U)$.

We proceed as above with the functor Pic. Keeping the previous notation of this section the isomorphism $f: S \rightarrow T$ which extends $\sigma$ induces an isomorphism from $\operatorname{Pic}(S)$ to $\operatorname{Pic}(T)$ by $f(|E|)=$ $\left|T \otimes_{S} E\right|$ for each class $|E|$ in $\operatorname{Pic}(S)$. Now let $|E| \in \operatorname{Pic}\left(S^{n}\right)$, then

$$
\begin{aligned}
J_{n-1}\left(f^{n}(|E|)\right) & =\Delta_{n-1}\left(\left|T^{n} \bigotimes_{S^{n}} E\right|\right) \\
& =\prod_{i=1}^{n+1}\left|\operatorname{Pic}\left(\varepsilon_{i}\right)\left(T^{n} \bigotimes_{S^{n}} E\right)\right|^{(-1)^{i-1}} \\
& =\prod_{i=1}^{n+1}\left|T_{i}^{n+1} \bigotimes_{T^{n}}\left(T^{n} \bigotimes_{S^{n}} E\right)\right|^{(-1)^{i-1}} \\
& =\prod_{i=1}^{n+1}\left|T_{i}^{n+1} \bigotimes_{S^{n}} E\right|^{(-1)^{i-1}} \\
& =\prod_{i=1}^{n+1}\left|T^{n+1} \bigotimes_{S^{n+1}} S_{i}^{n+1} \bigotimes_{S^{n}} E\right|^{(-1)^{2}-1} \\
& =\prod_{i=1}^{n+1} f^{n+1}\left|\operatorname{Pic}\left(\varepsilon_{i}\right)(E)\right|^{(-1)^{i-1}} \\
& =f^{n+1} \Delta_{n}(|E|) .
\end{aligned}
$$

In this calculation $S_{2}^{n+1}$ is an $S^{n}$-algebra via $\varepsilon_{i}$ and $T_{i}^{n+1}$ is a $T^{n}$-algebra by $\varepsilon_{i}$. Also $T^{n+1}$ is an $S^{n+1}$-algebra by $f^{n+1}$. It now follows exactly as in case " $U$ " above that $\sigma$ induces an isomorphism from $H^{n}\left(S / R\right.$, Pic) to $H^{n}(T / R$, Pic) and that $\operatorname{Aut}(R: S)$ acts on $H^{n}\left(S / R\right.$, Pic). Moreover, Aut $(R)$ acts on $\lim H^{n}(S / R, \mathrm{Pic})$ where the limit is taken over all étale extensions $S \overrightarrow{\text { of }} R$ so $\operatorname{Aut}(R)$ acts on $H_{\iota t}^{n}(R, \mathrm{Pic})$.

THEOREM 3. The natural monomorphism (given for example in [17]) from $B(R)$ to $H_{e t}^{2}(R, U)$ commutes with the action of $\operatorname{Aut}(R)$.

Proof. First, following [17] pg. 153, we describe $\lambda$. Let $A$ be 
an Azumaya $R$-algebra, and let $S$ be an étale $R$-algebra such that $|A| \in B(S / R)$. Then $A \otimes S \cong \operatorname{Hom}_{S}(P, P)$ for some $S$-progenerator $P$. Define $\phi: \mathrm{Hom}_{S \otimes S}(S \otimes P) \rightarrow \mathrm{Hom}_{S \otimes S}(P \otimes S)$ by the commutative diagram

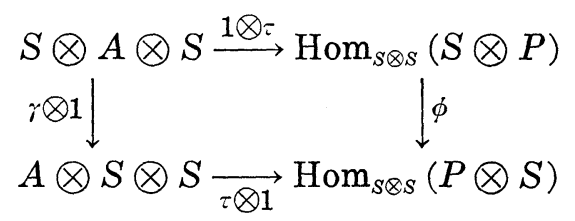

where $\tau$ is the given isomorphism from $A \otimes S$ to $\operatorname{Hom}_{S}(P, P)$ and $\gamma$ is the switch map. From the Morita theorem (Proposition 3.3, pg. 19 of [9]) $\phi$ is induced by an $S \otimes S$-isomorphism $S \otimes P \rightarrow$ $(P \otimes S) \otimes_{S \otimes S} I$ where $|I| \in \operatorname{Pic}(S \otimes S)$. By Proposition 13.13 of [15] we can, by extending $S$, assume $I \cong S \otimes S$ so $\phi$ is induced by an isomorphism $\rho: S \otimes P \rightarrow P \otimes S$. The isomorphism $\rho$ induces three $S \otimes S \otimes S$-isomorphisms: $\rho_{1}: S \otimes S \otimes P \rightarrow S \otimes P \otimes S, \rho_{2}: S \otimes S \otimes P \rightarrow$ $P \otimes S \otimes S$, and $\rho_{3}: S \otimes P \otimes S \rightarrow P \otimes S \otimes S$ where $\rho_{2}=\rho_{3} \rho_{1}$. Thus $\rho_{2} \rho_{3} \rho_{1}$ is multiplication by a unit $u(\tau, \rho)$ in $S \otimes S \otimes S$. The correspondence $A \rightarrow u(\tau, \rho)$ induces the monomorphism $\lambda$ from $B(R)$ into $H_{e t}^{2}(R, U)$. Given $S$ as above and $\sigma \in \operatorname{Aut}(R)$ we have an action of $\sigma$ from $H^{2}(S / R, U)$ to $H^{2}\left({ }_{\sigma} S / R, U\right)$ and it suffices to compare the image of $\left|{ }_{\sigma} A\right|$ in $H^{2}\left({ }_{\sigma} S / R, U\right)$ with $f^{3}(u(\tau, \rho))$. Now ${ }_{\sigma} A \otimes{ }_{\sigma} S \cong$ ${ }_{\sigma}(A \otimes S) \stackrel{\tau}{\cong}{ }_{\sigma} \operatorname{Hom}_{S}(P, P) \cong \operatorname{Hom}_{\sigma}{ }_{\sigma}\left({ }_{\sigma} P,{ }_{\sigma} P\right)$ and the composition of these isomorphisms is $\tau$. The diagram corresponding to (4) for ${ }_{\sigma} A$ is

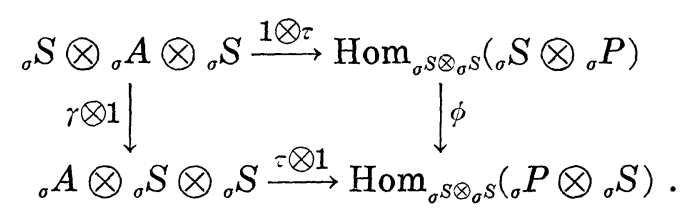

One can check that the $S \otimes S$ isomorphism $\rho: S \otimes P \rightarrow P \otimes S$ which gave rise to $\phi$ in (4) also is an ${ }_{\sigma} S \otimes{ }_{\sigma} S$ isomorphism from ${ }_{\sigma} S \otimes{ }_{\sigma} P$ to ${ }_{\sigma} P \otimes{ }_{\sigma} S$ which gives rise to $\phi$ in (5) (same $\phi !$ ). Therefore, $\rho_{2}^{-1} \rho_{3} \rho_{1}$ is multiplication by the unit $f^{3}(u(\tau, \rho))$ in ${ }_{\sigma} S \otimes{ }_{\sigma} S \otimes{ }_{\sigma} S$ on ${ }_{\sigma} S \otimes{ }_{\sigma} S \otimes$ ${ }_{\sigma} P$. That is, for any $x \in{ }_{o} S \otimes{ }_{o} S \otimes{ }_{\sigma} P, \quad \rho_{2}^{-1} \rho_{3} \rho_{1}(x)=u(\tau, \rho) x=$ $f^{3}(u(\tau, \rho)) \cdot x$. Therefore, ${ }_{\sigma} A$ corresponds to $f^{3}(u(\tau, \rho))$ in ${ }_{\sigma} S \otimes{ }_{\sigma} S \otimes$ ${ }_{\sigma} S$ which proves the theorem.

Let $S$ be a commutative finitely generated projective $R$-algebra. There is an exact sequence of Amitsur cohomology due to Chase and Rosenberg [2] which is

$$
\begin{aligned}
0 \longrightarrow & H^{1}(S / R, U) \stackrel{\alpha}{\longrightarrow} \operatorname{Pic}(R) \stackrel{\beta}{\longrightarrow} H^{0}(S / R, \mathrm{Pic}) \stackrel{d}{\longrightarrow} H^{2}(S / R, U) \\
& \stackrel{\gamma}{\longrightarrow}(S / R) \stackrel{\gamma}{\longrightarrow} H^{1}(S / R, \mathrm{Pic}) \stackrel{\rho}{\longrightarrow} H^{3}(S / R, U) .
\end{aligned}
$$


The maps in this sequence have been explicitly given in [16].

THEOREM 7. The homomorphisms in the exact sequence (6) of Chase and Rosenberg commute with the action of $\operatorname{Aut}(R: S)$.

Proof. Let $\sigma \in \operatorname{Aut}(R)$, let $S$ and $T$ be commutative finitely generated projective $R$-algebras and let $f$ be an extension of $\sigma$ from $S$ to $T$. Then we obtain the diagram

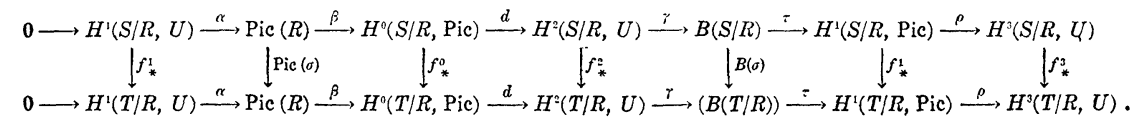

To prove the theorem it is sufficient that this diagram commutes. The method of proof is to write down each of the maps explicitly following [16] and to check the commutativity of the diagram on the cocycle level. This is a routine calculation. We carry out here only the proof of $B(\sigma) \gamma=\gamma f_{*}^{2}$. This will show the natural homomorphism $\gamma \cdot H^{2}(S / R, U) \rightarrow B(S / R)$ commutes with the action of $\operatorname{Aut}(R: S)$.

Let $u \in U(S \otimes S)$ be a 1-cocycle and let $E=\{x \in S \mid 1 \otimes x=x \otimes$ $1 \cdot u\}$. Then $E$ represents an element in Pic $(S / R)$ and $\alpha$ is induced by the correspondence $u \rightarrow E$. Now $f_{*}^{1}(|u|)=\left|f^{2}(u)\right|$ in $H^{1}(T / R, U)$. Let $F=\left\{x \in T \mid 1 \otimes x=x \otimes 1 \cdot f^{2}(u)\right\}$. Define $\psi:{ }_{\sigma} E \rightarrow F$ by $\psi(x)=$ $f(x)$ (note $\psi$ is well defined since ${ }_{\sigma} E=E$ as abelian groups) for $r \in R \quad$ and $\quad x \in{ }_{o} E ; \quad \psi(r * x)=\psi\left(\sigma^{-1}(r) x\right)=f\left(\sigma^{-1}(r) x\right)=r f(x)=r \psi(x)$. Thus $\alpha f_{*}^{1}(|u|)=\left|{ }_{\sigma} E\right|=\operatorname{Pic}(\sigma) \alpha(|u|)$.

Now $f^{3}(u)=\sum_{i} f\left(a_{i}\right) \otimes f\left(b_{i}\right) \otimes f\left(c_{i}\right) \in U\left(T^{3}\right)$. Let $Q=T \otimes T$ be the projective $T$-module obtained by letting $T$ act on the first factor. Define $T \otimes T$ isomorphism $h: T \otimes Q \rightarrow Q \otimes T$ by $h(x \otimes y \otimes z)=$ $\sum_{i} f\left(a_{i}\right) x \otimes f\left(c_{i}\right) z \otimes f\left(b_{i}\right) y$ for all $x, y, z$ in $T$. The isomorphism $h$ induces an isomorphism $\phi\left(f^{3}(u)\right): \operatorname{Hom}_{T \otimes T}(T \otimes Q, T \otimes Q) \rightarrow \operatorname{Hom}_{T \otimes T}(T \otimes Q$, $T \otimes Q)$ by $\phi\left(f^{3}(u)\right)(\rho)=h \rho h^{-1}$ for all $\rho \in \operatorname{Hom}_{T \& T}(T \otimes Q, T \otimes Q)$. The Azumaya algebra $A\left(f^{3}(u)\right)=\left\{w \in \operatorname{Hom}_{T}(Q, Q) \mid \phi\left(f^{3}(u)\right) \varepsilon_{1}(W)=\varepsilon_{2}(w)\right\}$ is a representative of the class $\gamma f_{*}^{2}(|u|)$ in $B(S / R)$. The algebra ${ }_{\sigma} A(u)$ represents the class $B(\sigma) \gamma(|u|)$ so it suffices to show $A\left(f^{3}(u)\right) \cong{ }_{\sigma} A$ as $R$-algebras. Now $f^{3}$ induces an isomorphism from $\mathrm{Hom}_{S \otimes S}(S \otimes P$, $S \otimes P)$ to $\mathrm{Hom}_{T \otimes T}(T \otimes Q, T \otimes Q)$ by $\rho \rightarrow f^{3} \rho\left(f^{3}\right)^{-1}$ for all $\rho \in$ $\mathrm{Hom}_{S \otimes, S}(S \otimes P, S \otimes P)$, and $f^{2}$ induces an isomorphism $\psi$ from $\operatorname{Hom}_{S}(P, P) \rightarrow \operatorname{Hom}_{T}(Q, Q)$ by $\psi(w)=f^{2} w\left(f^{2}\right)^{-1}$ for all $w \in \operatorname{Hom}_{S}(P, P)$. We show $\psi$ is a ring isomorphism from $A(u)$ to $A\left(f^{3}(u)\right)$. Let $w \in$ $A(u)$. We need to check that $\psi(w) \in A\left(f^{3}(u)\right)$. But 


$$
\begin{aligned}
\phi\left(f^{3}(u)\right) \varepsilon_{1}(\psi(w))= & \phi\left(f^{3}(u)\right) \varepsilon_{1}\left(f^{2} w\left(f^{2}\right)^{-1}\right) \\
= & \phi\left(f^{3}(u)\right) f^{3} \varepsilon_{1}(w)\left(f^{3}\right)^{-1} \\
= & h\left(f^{3} \varepsilon_{1}(w)\left(f^{3}\right)^{-1}\right) h^{-1} \\
= & \tau f^{3}(u) f^{3} \varepsilon_{1}(w)\left(f^{3}\right)^{-1} f^{3}(u)^{-1} \tau \\
& \text { (where } \tau \text { is twist map which exchanges } \\
& \text { the last two factors) } \\
= & \tau f^{3} u \varepsilon_{1}(w) u^{-1}\left(f^{3}\right)^{-1} \tau \\
= & f^{3} \tau u \varepsilon_{1}(w) u^{-1} \tau\left(f^{3}\right)^{-1} \\
= & f^{3} g \varepsilon_{1}(w) g^{-1}\left(f^{3}\right)^{-1} \\
= & f^{3} \phi(u) \varepsilon_{1}(w)\left(f^{3}\right)^{-1} \\
= & f^{3} \varepsilon_{2}(w)\left(f^{3}\right)^{-1} \\
= & \varepsilon_{2}\left(f^{2}(w)\left(f^{2}\right)^{-1}\right) \\
= & \varepsilon_{2}(\psi(w))
\end{aligned}
$$

For any $r \in R$ and $w \in{ }_{\sigma} A(u)$ we have $\psi(r * w)=f^{2} \sigma^{-1}(r) w\left(f^{2}\right)^{-1}=$ $r f^{2} w\left(f^{2}\right)^{-1}=r \psi(w)$ so $\psi$ is an $R$-isomorphism from ${ }_{o} A(u)$ to $A\left(f^{3}(u)\right)$.

CoRollary 9. If $S$ is a commutative finitely generated projective $R$-algebra and $\operatorname{Pic}(S)=\operatorname{Pic}(S \otimes S)=0$ then $\gamma$ is a natural Aut $(R: S)$ isomorphism from $H^{2}(S / R, U)$ to $B(S / R)$.

Corollary 10. Let $S$ be a Galois extension of $R$, and assume $S$ has no idempotents other than 0 and 1 . Let $G$ be the Galois group of $S$ over $R$. Then the homomorphisms in the seven-term sequence of Galois cohomology

$$
\begin{gathered}
1 \longrightarrow H^{1}(G, U(S)) \longrightarrow \operatorname{Pic}(R) \longrightarrow \operatorname{Pic}(S)^{G} \longrightarrow H^{2}(G, U(S)) \\
\longrightarrow B(S / R) \longrightarrow H^{1}(G, \operatorname{Pic}(S)) \longrightarrow H^{3}(G, U(S))
\end{gathered}
$$

commute with the action of Aut $(R: S)$. In particular, if Pic $(S)=0$ then $H^{2}(G, U(S))$ is isomorphic to $B(S / R)$ by an isomorphism which commutes with the action of $\operatorname{Aut}(R: S)$.

Proof. If $S$ is a Galois extension of $R$ with group $G$ and $S$ has no idempotents other than 0 and 1 then $\operatorname{Aut}_{R}(S)=G$. Thus the group $\mathscr{G}$ of automorphism of $S$ leaving $R$ setwise fixed acts on $G$ by conjugation. If $\sigma \in \mathscr{G}$ and $\tau \in G$ we let $\tau^{\sigma}=\sigma^{-1} \tau \sigma$. Assume $M$ is a $\mathscr{G}$-module. Let $f \in Z^{n}(G, M)$ be a Galois $n$-cocycle and let $\sigma f \in Z^{n}(G, M)$ be defined by $\sigma f\left(\tau_{1}, \cdots, \tau_{n}\right)=\sigma\left[f\left(\tau_{1}^{o}, \cdots, \tau_{n}^{o}\right)\right]$ for $\tau_{i} \in G$ and $n \geqq 1$ and let $\sigma \cdot m=\sigma(m)$ for $m \in Z^{\circ}(G, M)$. Then a direct calculation gives $\partial_{n} \sigma \cdot f=\sigma \cdot \partial_{n} f$ for all $n \geqq 0$. Thus $\mathscr{G}$ acts on $H^{n}(G, M)$ with the action induced by the correspondence $f \rightarrow \sigma \cdot f$. 
By a tedious calculation with cocycles one can check that if $\sigma \in G$ then $f$ and $\sigma f$ represent the same class in $H^{n}(G, M)$. It follows that Aut $(R: S)$ acts on $H^{n}(G, U(S))$ and $H^{n}(G$, Pic $(S))$ for all $n \geqq 0$. This action is given in [11] where these assertions are verified when $S$ is a field. Proofs are the same in the general context.

Next we check following notation in [15], p. 120-125, that the actions we have defined on $H^{n}(S / R, F)$ and $H^{n}(G, F)$ correspond to one another. The isomorphisms $\phi_{n}: S^{n+1} \rightarrow K^{n}(G, S)$ given by $\dot{\rho}_{n}\left(s \otimes \cdots \otimes s_{n}\right)\left(\tau_{k}, \cdots, \tau_{n}\right)=s_{1} \tau_{1}\left(s_{2}\right) \tau_{1} \tau_{2}\left(s_{3}\right) \cdots \tau_{1} \cdots \tau_{n}\left(s_{n+1}\right)$ for $s_{i} \in S$ and $\tau_{i} \in G$ induce a homomorphism $F\left(\phi_{n}\right): F\left(S^{n+1}\right) \rightarrow F\left(K^{n}(G, S)\right)=K^{n}(G, F(S))$ which induces a morphism of complexes for any additive functor $F$. This morphism of complexes induces homomorphisms $\psi_{n}: H^{n}(S / R$, $F) \rightarrow H^{n}(G, F(S))$. Let $\sigma \in G$, then

$$
\begin{aligned}
\sigma \dot{\phi}_{n}\left(s_{1} \otimes \cdots \otimes s_{n}\right)\left(\tau_{1}, \cdots, \tau_{n}\right) & =\sigma\left[s_{1} \tau_{1}^{\sigma}\left(s_{2}\right) \cdots \tau_{1}^{\sigma} \cdots \tau_{n}^{\sigma}\left(s_{n+1}\right)\right] \\
& =\sigma\left(s_{1}\right) \tau_{1} \sigma\left(s_{2}\right) \cdots \tau_{1} \cdots \tau_{n} \sigma\left(s_{n+1}\right) \\
& =\phi_{n} \sigma\left(s_{1} \otimes \cdots \otimes s_{n+1}\right) .
\end{aligned}
$$

Thus if $F$ is the units functor $U$ then the induced homomorphism $i_{n}: H^{n}(S / R, U) \rightarrow H^{n}(G, U(S))$ is an $\operatorname{Aut}(R: S)$ homomorphism. In a similar way the induced homomorphism $\psi_{n}: H^{n}(S / R, \mathrm{Pic}) \rightarrow H^{n}(G$, Pic $(S))$ can be checked to be Aut $(R: S)$ homomorphisms since Pic $\left(\phi_{n}\right)$ : Pic $\left(S^{n+1}\right) \rightarrow$ Pic $\left(K^{n}(G, S)\right)$ is an Aut $(R: S)$ isomorphism.

Now the result is a consequence of Theorem 7 and Corollary 5.5 of [1] which connects the exact sequence of Amitsur cohomology with the exact sequence of Galois cohomology.

We saw before that Aut $(R)$ acts on $H_{e t}^{n}(R, U)$. We now expose the corresponding result in Galois cohomology. Let $R$ be a commutative ring with no idempotents other than 0 and 1 and let $\Omega$ be the separable closure of $R$ (see p. 99 of [9]). For any $\sigma \in \operatorname{Aut}(R)$ it is easy to check that ${ }_{\sigma} \Omega$ is another separable closure of $R$. By uniqueness of the separable closure we know $\Omega \cong{ }_{\sigma} \Omega$ as $R$-algebras. By Lemma 1e, it follows that $\operatorname{Aut}(R)=\operatorname{Aut}(R: \Omega)$. Let $S$ be a Galois extension of $R$ in $\Omega$, then ${ }_{\sigma} S$ is a Galois extension of $R$ in $\Omega$ and if $G$ is the Galois group of $S$ then $G$ is also the Galois group of ${ }_{\sigma} S$. Let $f$ be an extension of $\sigma$ from $S$ to ${ }_{\sigma} S$ and let $g$ be an $n$-cocycle in $Z^{n}(G, U(S))$. There is a corresponding $n$-cocycle $h$ in $Z^{n}\left(G, U\left({ }_{o} S\right)\right)$ by $h\left(\tau_{1}, \cdots, \tau_{n}\right)=f\left(g\left(f^{-1} \tau_{1} f, \cdots, f^{-1} \tau_{n} f\right)\right)$. As with Amitsur cohomology $\sigma$ induces an isomorphism from $H^{n}(G, U(S))$ to $H^{n}\left(G, U\left({ }_{\sigma} S\right)\right)$. This action is compatible with the maps in the direct limit system $\lim H^{n}(G, U(S))$ where the limit is taken over all Galois extensions $S$ of $R$ in $\Omega$. Thus Aut $(R)$ acts on $H_{\text {\&:1 }}^{n}(R, U)$, and this action commutes with the natural homomorphism from $H_{\text {Gal }}^{2}(R, U)$ to $B(R)$. 
Let $\mathscr{G}$ be the subgroup of Aut $(A)$ which leaves $R \cdot 1$ setwise invariant. If Center $A=R \cdot 1$ then $\mathscr{G}=\operatorname{Aut}(A)$. If $A$ is a faithful $R$-algebra and $\tau$ is the restriction map from $\mathscr{G}$ to $\operatorname{Aut}(R)$ then from Lemma le the sequence

$$
1 \longrightarrow \operatorname{Aut}_{R}(A) \stackrel{i}{\longrightarrow} \mathscr{G} \stackrel{r}{\longrightarrow} \operatorname{Aut}(R: A) \longrightarrow 1
$$

is exact.

Now the splitting of the exact sequence (11) over the finite subgroups $H \subset$ Aut $(R: A)$ for an Azumaya $R$-algebra is considered. The given sequence splits over $H$ in case there is a monomorphism $s: H \rightarrow \operatorname{Aut}(A)$ such that $r s=1_{H}$.

Theorem 12. Let $A$ be an Azumaya $R$-algebra, let $H$ be a finite subgroup of $\operatorname{Aut}(R: A)$, and let $K=\{r \in R \mid \tau(r)$ for all $\tau \in H\}$. Assume $R$ is a Galois extension of $K$ with group $H$, then the following are equivalent.

(1) The sequence (11) splits over $H$.

(2) There is an Azumaya K-algebra $B$ such that $A \cong R \bigotimes_{K} B$ as $R$-algebras.

(3) $A$ is a normal algebra and the class of $A$ in $B(R)$ is in the kernel of the Teichmuller cocycle map [3].

Proof. If $A \cong R \bigotimes_{K} B$ then each element $\sigma \in H$ induces the $K$-automorphism $\sigma \otimes 1$ of $A$ and the splitting map is $s(\sigma)=\sigma \otimes 1$ so $2 \rightarrow 1$. Next assume the existence of a splitting map $s$. Then there is a group of automorphisms $H^{\prime}$ of $A$ whose restriction to $R$ is $H$. In this case all the hypotheses of Lemma 2 of [6] are satisfied. Let $B=\left\{a \in A \mid \sigma(a)=a\right.$ for all $\left.\sigma \in H^{\prime}\right\}$. In the proof of Lemma 2 of [6] it is shown that $B$ is an Azumaya $K$-algebra and $R \bigotimes_{K} B \cong A$ by $r \otimes b \rightarrow r b$ for all $r \in R, b \in B$ so $1 \rightarrow 2$. An Azumaya $R$-algebra $A$ is called normal if $H$ is a subgroup of $\operatorname{Aut}(R: A)$. By Corollary 5.2 of [3], the class in $B(R)$ represented by a normal algebra $A$ has a trivial image under the Teichmuller cocycle map if and only if there is an Azumaya $K$-algebra $B$ such that $A \cong R \bigotimes_{K} B$ as $R$ algebras. Thus $2 \leftrightarrow 3$.

We saw in Lemma 1 that if $\sigma \in \operatorname{Aut}(R)$ then $\sigma$ extends to an automorphism of the Azumaya algebra $A$ if and only if $A \cong{ }_{0} A$ as $R$-algebras. We have let $\operatorname{Aut}(R: A)=\left\{\sigma \in \operatorname{Aut}(R) \mid A \cong{ }_{\sigma} A\right\}$. When does $\operatorname{Aut}(R: A)=\left\{\sigma \in \operatorname{Aut}(R) \| A|=|{ }_{\sigma} A \mid\right\}$ ? We first have the following positive result.

Proposition 13. Let $R$ denote a local ring or the ring of polynomials in one variable over a perfect field. For any Azumaya 
$R$-algebra $A$ and any $\sigma \in \operatorname{Aut}(R)$ we have $A \cong{ }_{0} A$ as $R$-algebras if and only if the class of $A$ and ${ }_{0} A$ are the same in $B(R)$.

Proof. If $A \cong{ }_{\sigma} A$ as $R$-algebras then $|A|=\left|{ }_{\sigma} A\right|$. Conversely, Corollary 1 of [7] implies $A \cong M_{n}(D)$ where $D$ is the unique Azumaya $R$-algebra with no idempotents other than 0 and 1 in the same class as $A$ in $B(R)$. Employing Lemma 1 we have the chain of isomorphisms, ${ }_{\sigma} A \cong{ }_{\sigma}\left(D \otimes \operatorname{Hom}_{R}\left(R^{n}, R^{n}\right)\right) \cong{ }_{\sigma} D \otimes \operatorname{Hom}_{R}\left({ }_{\sigma} R^{n},{ }_{\sigma} R^{n}\right) \cong M_{n}\left({ }_{\sigma} D\right)$. If $|A|=\left|{ }_{\sigma} A\right|$ then by the uniqueness of $D$ we have $D \cong{ }_{\sigma} D$ so $A \cong{ }_{\sigma} A$.

Now we outline an example which shows the previous proposition fails even when $R$ is a Dedekind domain. Let $R$ be a Dedekind domain and let $I$ be a fractional $R$-ideal. Let $M=R \oplus I$ and $A=$ $\operatorname{Hom}_{R}(M, M)$. Then $A$ is an Azumaya $R$-algebra. Let $\sigma \in \operatorname{Aut}(R)$, then ${ }_{\sigma} A \cong \operatorname{Hom}_{R}\left({ }_{\sigma} M,{ }_{\sigma} M\right)$ by Lemma 1. By Moritia's theory ( $\# 7$, pg. 37 of [9]) we have $A \cong{ }_{\sigma} A$ if and only if ${ }_{\sigma} M \cong J \otimes M$ for some fractional $R$-ideal $J$.

But ${ }_{o} M \cong R \oplus{ }_{o} I$ and $J \otimes M \cong R \oplus I J^{2}$, so $M \cong{ }_{\sigma} M$ if and only if there is a fractional $R$-ideal $J$ so that ${ }_{\sigma} I=I J^{2}$. Now $A$ is always equivalent to ${ }_{\sigma} A$ in $B(R)$ and so to give a counter example to the conclusion of Proposition 13 if $R$ is a Dedekind domain it suffices to give a Dedekind domain $R$ such that Pic $(R)$ is the group of order 4 and exponent 2 and so that Aut $R$ acts nontrivially on Pic $(R)$. Such an example is easy to construct using results of $\mathrm{L}$. Claborn [5]. It follows that under the action of $\operatorname{Aut}(R)$ on $B(R)$ we have $B(R)^{G}$ is guaranteed to consist of those classes in $B(R)$ represented by normal algebras only when the hypotheses of Proposition 13 are satisfied.

Proposition 14. Let $K$ be a field of characteristic $=0 . \quad$ Then the Schur subgroup is an Aut $(K)$ invariant subgroup of $B(K)$.

Proof. Let $K$ be a field of characteristic $=0$. A cyclotomic algebra is a crossed product over $K$ of the form $\Delta(K \sqrt[n]{1}, G, f)$ where $f$ is a 2-cocycle on $G$ with its values in the cyclic group generated by $\sqrt[n]{1}$. The Schur subgroup of $B(K)$ consists of those classes in $B(K)$ represented by a cyclotomic algebra [19]. If $\sigma \in$ Aut $(K)$ then $\sigma \in \operatorname{Aut}(K: K \sqrt[n]{1})$ and ${ }_{\sigma} \Delta(K \sqrt[n]{1}, G, f)=\Delta(K \sqrt[n]{1}, G$, $\sigma \cdot f)$. But $\sigma \cdot f(\tau, \rho)=\sigma\left[f\left(\tau^{\sigma}, \rho^{\sigma}\right)\right]$ so the values of $\sigma \cdot f$ are in the $n$th roots of unity and $\Delta(K \sqrt[n]{1}, G, \sigma \cdot f)$ is another cyclotomic algebra. Thus Aut $(R)$ leaves the Schur subgroup of $B(K)$ invariant.

Finally, let $R=\boldsymbol{R}[x, y] /(x y-1)$ where $\boldsymbol{R}$ denotes the real numbers. Then $B(R)=Z_{2} \oplus Z_{2}[10]$. Let $S=C \otimes_{R} R$, then $S$ is a 
Galois extension of $R$ with Galois group $G$ of order 2 with generator of $G$ denoted $\tau$. Let $\sigma \in \operatorname{Aut}(R)$ be given by $\sigma(x)=-x$ and $\sigma(y)=$ $-y$. Let $\Delta(S: G: f)$ be the crossed product with $f(\tau, \tau)=x$. Then $\Delta(S: G: f)$ represents an element in $B(R)$ of order 2 and ${ }_{\sigma} \Delta(S: G: f)=$ $\Delta(S: G: \sigma \cdot f)$ where $\sigma \cdot f(\tau, \tau)=\sigma(x)=-x$. Thus ${ }_{\sigma} \Delta(S: G: f)$ represents an element in $B(R)$ inequivalent to $\Delta(S: G: f)$. The crossed product $A(S: G: g)$ with $g(\tau, \tau)=-1$ represents a nontrivial element in $B(R)$ such that ${ }_{\sigma} \Delta(S: G: g) \cong \Delta(S: G: g)$ for all $\sigma \in B(R)$ so the image of Aut $(R)$ in Aut $(B(R))$ has order 2. Not every automorphism of $B(R)$ can be represented by an automorphism of $R$.

\section{REFERENCES}

1. S. U. Chase, D. K. Harrison and A. Rosenberg, Galois theory and cohomology of commutative rings, Hem. Amer. Math. Soc., 52 (1968), 15-33.

2. S. U. Chase and A. Rosenberg, Amitsur cohomology and the Brauer group, Mem. Amer. Math. Soc., 52 (1968), 20-65.

3. L. N. Childs, On Normal Azumaya algebras and the Teichmuller cocycle map, J. Algebra, 23 (1972), 1-17.

4. - Mayer-Vietoris sequences and Brauer groups of nonnormal domains, Trans. Amer. Math. Soc., 196 (1974), 51-67.

5. L. Claborn, Every abelian group is a class group, Pacific J. Math., 18 (1966), 219-222.

6. F. R. DeMeyer, Some notes on the general Galois theory of rings, Osaka J. Math., 2 (1965), 117-127.

7. — Projective modules over central separable algebras, Canad. J. Math., 21 (1969), 39-43.

8. The Brauer group of a ring modulo an ideal, Rocky Mtn. J. Math., 6 (1976), 191-198.

9. F. R. DeMeyer and E. Ingraham, Separable algebras over commutative rings, Lecture Notes in Mathematics 181, Springer-Verlag, New York, 1971.

10. F. R. DeMeyer and M. A. Knus, The Brauer group of a real curve, P.A.M.S., 57 (1976), 227-232.

11. S. Eilenberg and S. MacLane, Cohomology and Galois theory I, Normality of algebras and Teichmuller's cocycle, Trans. Amer. Math. Soc., 64 (1948), 1-20.

12. O. Gabber, Some theorems on Azumaya algebras, Ph. D. Thesis, Harvard U. (1978).

13. G. J. Janusz, Automorphism groups of simple algebras and group algebras, Proc. of the Philadelphia Conf. on Ring Theory, M. Dekker. 1979.

14. M. A. Knus and M. Ojanguren, Théorie de la Déscente et Algèbres d'Azumaya, Lecture Notes in Mathematics 389, Springer-Verlag, New York, 1974.

15. - A Mayer-Vietoris sequence for the Brauer group, J. Pure and Appl. Algebra, 5 (1974), 345-360.

16. M. A. Knus, A Teichmuller cocycle for finite extensions, (Preprint) E.T.H. Zurich, (1975).

17. M. Orzech and C. Small, The Brauer group of commutative rings, Lecture Notes in Pure and Applied Mathematics, 11 Marcel Dekker (1975).

18. B. Pareigis, Uber normale, zentrale, separable Algebren und Amitsur-Kohomologie, Math. Annalen, 154 (1964), 330-340.

19. T. Yamada, The Schur subgroup of the Brauer group, Lecture Notes in Mathematics 397, Springer-Verlag, New York, 1974. 
Received October 7, 1980.

Colorado State University

FORT COLlins, CO 80523 


\section{PACIFIC JOURNAL OF MATHEMATICS}

EDITORS

DONALD BABBITT (Managing Editor)

University of California

Los Angeles, CA 90024

Hugo RossI

University of Utah

Salt Lake City, UT 84112

C. C. MOORE and ANDREW OGG

University of California

Berkeley, CA 94720

\section{J. DugundjI}

Department of Mathematics

University of Southern California

Los Angeles, CA 90007

R. FINN and J. MILGRAM

Stanford University

Stanford, CA 94305

\section{ASSOCIATE EDITORS}
R. ARENS
E. F. BECKENBACH
B. H. NEUManN
F. WOLF
K. YoSHIDA

\section{SUPPORTING INSTITUTIONS}

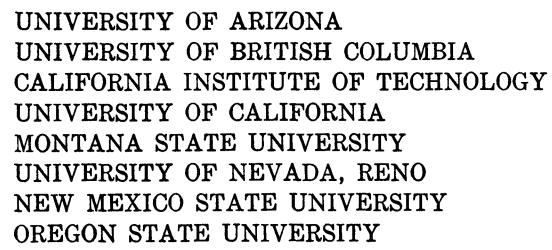

UNIVERSITY OF ARIZONA

UNIVERSITY OF BRITISH COLUMBIA

CALIFORNIA INSTITUTE OF TECHNOLOGY

UNIVERSITY OF CALIFORNIA

MONTANA STATE UNIVERSITY

UNIVERSITY OF NEVADA, RENO

NEW MEXICO STATE UNIVERSITY

OREGON STATE UNIVERSITY

\author{
UNIVERSITY OF OREGON \\ UNIVERSITY OF SOUTHERN CALIFORNIA \\ STANFORD UNIVERSITY \\ UNIVERSITY OF HAWAII \\ UNIVERSITY OF TOKYO \\ UNIVERSITY OF UTAH \\ WASHINGTON STATE UNIVERSITY \\ UNIVERSITY OF WASHINGTON
}

The Supporting Institutions listed above contribute to the cost of publication of this Journal, but they are not owners or publishers and have no responsibility for its content or policies.

Mathematical papers intended for publication in the Pacific Journal of Mathematics should be in typed form or offset-reproduced, (not dittoed), double spaced with large margins. Please do not use built up fractions in the text of the manuscript. However, you may use them in the displayed equations. Underline Greek letters in red, German in green, and script in blue. The first paragraph or two must be capable of being used separately as a synopsis of the entire paper. Please propose a heading for the odd numbered pages of less than 35 characters. Manuscripts, in triplicate, may be sent to any one of the editors. Please classify according to the scheme of Math. Reviews, Index to Vol. 39. Supply name and address of author to whom proofs should be sent. All other communications should be addressed to the managing editor, or Elaine Barth, University of California, Los Angeles, California, 90024.

50 reprints to each author are provided free for each article, only if page charges have been substantially paid. Additional copies may be obtained at cost in multiples of 50 .

The Pacific Journal of Mathematics is issued monthly as of January 1966. Regular subscription rate: $\$ 102.00$ a year (6 Vols., 12 issues). Special rate: $\$ 51.00$ a year to individual members of supporting institutions.

Subscriptions, orders for numbers issued in the last three calendar years, and changes of address shoud be sent to Pacific Journal of Mathematics, P.O. Box 969, Carmel Valley, CA 93924, U.S.A. Old back numbers obtainable from Kraus Periodicals Co., Route 100, Millwood, NY 10546.

\section{PUBLISHED BY PACIFIC JOURNAL OF MATHEMATICS, A NON-PROFIT CORPORATION}

Printed at Kokusai Bunken Insatsusha (International Academic Printing Co., Ltd.). 8-8, 3-chome, Takadanobaba, Shinjuku-ku, Tokyo 160, Japan. 


\section{Pacific Journal of Mathematics}

\section{Vol. 97, No. $2 \quad$ February, 1981}

Patrick Robert Ahern and N. V. Rao, A note on real orthogonal measures . . . . . 249

Kouhei Asano and Katsuyuki Yoshikawa, On polynomial invariants of fibered

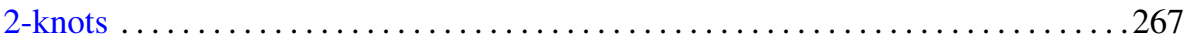

Charles A. Asmuth and Joe Repka, Tensor products for $S L_{2}(\mathscr{K})$. I.

Complementary series and the special representation

Gary Francis Birkenmeier, Baer rings and quasicontinuous rings have a

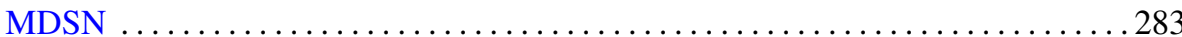

Hans-Heinrich Brungs and Günter Törner, Right chain rings and the generalized

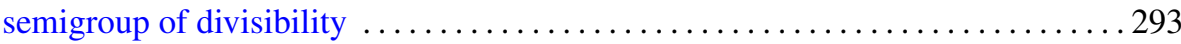

Jia-Arng Chao and Svante Janson, A note on $H^{1} q$-martingales . . . . . . . . . 307

Joseph Eugene Collison, An analogue of Kolmogorov's inequality for a class of

additive arithmetic functions

Frank Rimi DeMeyer, An action of the automorphism group of a commutative ring on its Brauer group

H. P. Dikshit and Anil Kumar, Determination of bounds similar to the Lebesgue

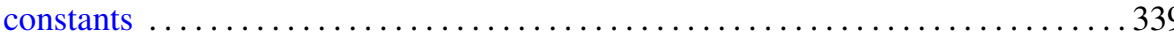

Eric Karel van Douwen, The number of subcontinua of the remainder of the plane

D. W. Dubois, Second note on Artin's solution of Hilbert's 17th problem. Order spaces

Daniel Evans Flath, A comparison of the automorphic representations of GL(3) and its twisted forms

Frederick Michael Goodman, Translation invariant closed $*$ derivations

Richard Grassl, Polynomials in denumerable indeterminates

K. F. Lai, Orders of finite algebraic groups

George Kempf, Torsion divisors on algebraic curves

Arun Kumar and D. P. Sahu, Absolute convergence fields of some triangular matrix methods

Elias Saab, On measurable projections in Banach spaces

Chao-Liang Shen, Automorphisms of dimension groups and the construction of AF algebras

Barry Simon, Pointwise domination of matrices and comparison of $\Phi_{p}$ norms

Chi-Lin Yen, A minimax inequality and its applications to variational inequalities

Stephen D. Cohen, Corrections to: "The Galois group of a polynomial with two indeterminate coefficients"

Phillip Schultz, Correction to: "The typeset and cotypeset of a rank 2 abelian group"

Pavel G. Todorov, Correction to: "New explicit formulas for the $n$th derivative of composite functions"

Douglas S. Bridges, Correction to: "On the isolation of zeroes of an analytic function" 